JOURNAL OF SYNCHROTRON RADIATION

ISSN 1600-5775

Received 1 March 2018

Accepted 23 July 2018

Edited by Y. Amemiya, University of Tokyo, Japan

Keywords: high-energy surface X-ray diffraction (HESXRD); planar laser-induced fluorescence (PLIF); CO oxidation; $\operatorname{Pd}(100)$.

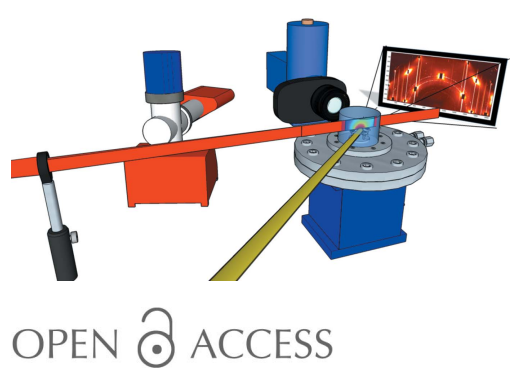

\section{Combining synchrotron light with laser technology in catalysis research}

\author{
Sara Blomberg, ${ }^{\text {a* }}$ Johan Zetterberg, ${ }^{\mathrm{b}}$ Johan Gustafson, ${ }^{\mathrm{a}}$ Jianfeng Zhou, \\ Mikhail Shipilin, ${ }^{\text {a }}$ Sebastian Pfaff, ${ }^{\text {b }}$ Uta Hejral, ${ }^{a}$ Per-Anders Carlsson, ${ }^{c}$ \\ Olof Gutowski, ${ }^{d}$ Florian Bertram ${ }^{\mathrm{d}}$ and Edvin Lundgren ${ }^{\mathrm{a}}$
}

\footnotetext{
aSynchrotron Radiation Research, Lund University, Box 118, Lund 22100, Sweden, ${ }^{\mathbf{b}}$ Combustion Physics, Lund University, Box 118, Lund 22100, Sweden, 'Competence Centre for Catalysis, Chalmers University of Technology, Gothenburg 41296, Sweden, and dPhoton Science, DESY, Notkestrasse 85, Hamburg 22607, Germany.

*Correspondence e-mail: sara.blomberg@chemeng.Ith.se
}

High-energy surface X-ray diffraction (HESXRD) provides surface structural information with high temporal resolution, facilitating the understanding of the surface dynamics and structure of the active phase of catalytic surfaces. The surface structure detected during the reaction is sensitive to the composition of the gas phase close to the catalyst surface, and the catalytic activity of the sample itself may affect the surface structure, which in turn may complicate the assignment of the active phase. For this reason, planar laser-induced fluorescence (PLIF) and HESXRD have been combined during the oxidation of $\mathrm{CO}$ over a $\mathrm{Pd}(100)$ crystal. PLIF complements the structural studies with an instantaneous two-dimensional image of the $\mathrm{CO}_{2}$ gas phase in the vicinity of the active model catalyst. Here the combined HESXRD and PLIF operando measurements of $\mathrm{CO}$ oxidation over $\mathrm{Pd}(100)$ are presented, allowing for an improved assignment of the correlation between sample structure and the $\mathrm{CO}_{2}$ distribution above the sample surface with sub-second time resolution.

\section{Introduction}

Catalysis is widely used in the industrial production of, for instance, base and speciality chemicals and fuels but is also used in the reduction of toxic emissions from stationary and mobile sources.

The catalyst accelerates a chemical reaction by providing an alternative pathway with a lower-energy barrier for the reaction, without being consumed. The heterogeneously catalyzed pathway often proceeds via adsorption of the reactants from a fluid phase onto the surface of a solid catalyst, which mediates the catalytic reaction. A common type of catalyst is the socalled supported catalyst, which often consists of catalytically active nanoparticles embedded in a porous high-surface-area oxide. The complex composition of industrial catalysts and the harsh environmental conditions in the reactor make the feasibility for fundamental surface studies of the catalyst under operating conditions challenging. The details of a catalytic reaction are therefore, in most cases, not fully understood under realistic reaction conditions.

Simplified model systems with, for example, single crystals are therefore used to gain fundamental knowledge about the catalytically active surface (Ertl et al., 1997). The well defined surface of a single crystal enables the study of specific properties of surface sites or surface structures that are present on the industrial catalytic nanoparticle, and how they contribute to the catalytic activity (Westerström et al., 2007; Todorova et 
al., 2003; Mavrikakis et al., 1998; Hammer, 2006; Gustafson et al., 2005; Gao et al., 2009; Somorjai \& Li, 2010).

$\mathrm{CO}$ oxidation is considered one of the simplest reactions and is therefore traditionally chosen as a model reaction when surface-science studies are performed (Freund et al., 2011). The experiments are often performed ex situ or during exposures at low pressures, typically ranging from $10^{-10}$ mbar to $10^{-6}$ mbar. In this manner, the well defined model of the industrial catalyst can be investigated under well controlled conditions and reliable data can be obtained. Despite these previously well controlled ex situ studies, the active phase of $\mathrm{CO}$ oxidation on a Pd-based catalyst is still under debate (Gao et al., 2009, 2010; Rijn, Balmes et al., 2010). In operando studies, the catalyst is studied under working conditions such that the surface structure interacting with gas-phase molecules can be correlated to the function of the catalyst (Blomberg et al., 2013; Hendriksen et al., 2010; Ackermann et al., 2005; Gustafson et al., 2008; Gao et al., 2009; Toyoshima \& Kondoh, 2015; Lundgren et al., 2017; Chen et al., 2007; Rupprechter \& Weilach, 2007). To achieve a better understanding of the gas-surface interaction, the advantage of having experimental setups which allow the combination of several techniques have been highlighted in the last decade (Head et al., 2017; Tinnemans et al., 2006). Some of the advantages of collecting data with several techniques in the same setup are that the results can be achieved under the same conditions. In the ideal case, the techniques can operate simultaneously under operating conditions, giving the opportunity to reach a more detailed understanding of the processes under study.

The activity of the catalyst is often measured by studying the gas composition detected by a mass spectrometer (MS). When the MS is positioned at the outlet of the reactor, as in the present study, the result is a poor spatially resolved measurement and delayed detection of the gas-phase molecules, determined by the path length that the gas molecules travel before reaching the MS detector. For this purpose, we have used planar laser-induced fluorescence (PLIF) to probe the gas phase instantaneously and visualize it close to the catalyst surface (Blomberg et al., 2016). In the present study, PLIF has been used in combination with high-energy surface X-ray diffraction (HESXRD) for surface structure determination (Gustafson et al., 2014). By combining these techniques, we can link the surface structure to the $\mathrm{CO}_{2}$ production (detected $0.3 \mathrm{~mm}$ above the surface non-intrusively) on a subsecond scale. Our observations show that the position of the MS is crucial in determining a correct correlation between catalytic activity and the surface structure of the catalyst.

\section{Methods}

The experiments were performed at beamline P07 at PETRA III positioned at Deutsches Elektronen-Synchrotron (DESY). This beamline is designed for material studies with hard X-ray radiation (King et al., 2014). The high-energy $\mathrm{X}$-rays enable full surface-structure determination, where each detector image contains the projection of a large area of the reciprocal space, on a time scale suitable for operando studies (Gustafson et al., 2014; Shipilin et al., 2014, 2016; Hejral et al., 2016, 2017). The incident angle of the X-rays was set to $0.04^{\circ}$, close to the critical angle of $\mathrm{Pd}(100)$ at the photon energy used $(85 \mathrm{keV})$. The diffraction pattern was detected with a temporal resolution of $2 \mathrm{~Hz}$ by a two-dimensional Perkin-Elmer detector positioned $1.75 \mathrm{~m}$ from the sample. The detector was protected from high-intensity X-rays at the positions of the $\operatorname{Pd}(100)$ and the sapphire reactor walls' Bragg reflections by $\mathrm{W}$ pieces. These blocks are seen as dark rectangles in the diffraction images (Fig. 2).

In addition, a laser in the infrared spectral region $(2.7 \mu \mathrm{m})$ was used to probe $\mathrm{CO}_{2}$ in the gas phase in the vicinity $(0.3 \mathrm{~mm})$ of the $\operatorname{Pd}(100)$ surface at a repetition rate of $10 \mathrm{~Hz}$. PLIF is a species-specific technique, in which the molecule of interest is excited by a laser sheet and then relaxes by emission of a photon (i.e. fluorescence), and the fluorescence is detected using a camera. Our target molecule $\left(\mathrm{CO}_{2}\right)$ can be probed via ro-vibrational transitions in the mid-infrared. There are several detection schemes that can be employed for probing $\mathrm{CO}_{2}$, e.g. the overtone and combination band at around $2.0 \mu \mathrm{m}\left(12^{0} 1\right) \rightarrow\left(00^{0} 0\right)$ (Alwahabi et al., 2007), the combination band at $2.7 \mu \mathrm{m}\left(00^{\circ} 0\right) \rightarrow\left(10^{\circ} 1\right)$ (Zetterberg et al., 2012; Kirby \& Hanson, 2002) and the fundamental band at around $4.3 \mu \mathrm{m}$ (Goldenstein et al., 2015). In this study, the combination band was probed in the $\mathrm{CO}_{2}$ molecule using a pulsed laser at $\sim 2.7 \mu \mathrm{m}$ with a pulse duration of $\sim 5 \mathrm{~ns}$ and a power of $\sim 7 \mathrm{~mJ}$ pulse ${ }^{-1}$. The laser beam formed a thin laser sheet of $\sim 6 \mathrm{~mm}$ height by sheet-shaping optics and then sent through the sapphire reactor dome $\sim 0.3 \mathrm{~mm}$ above the sample surface. The $\mathrm{CO}_{2}$ fluorescence was then imaged onto a two-dimensional focal plane array (FPA) (SBF LP134, Santa Barbara Focal Plane) perpendicular to the laser sheet. The camera exposure time was set to $30 \mu$ s and chosen for efficient collection of the $\mathrm{CO}_{2}$ fluorescence signal while avoiding detector saturation by the thermal background. To address the varying thermal background, the FPA was triggered at $20 \mathrm{~Hz}$, thus taking an extra image between every laser shot, making subtraction of the thermal background possible on a singleshot basis. A more detailed description of the experimental setup and detection scheme can be found in previous work (Zhou et al., 2017). Images of the $\mathrm{CO}_{2}$ distribution were acquired every $0.1 \mathrm{~s}$, but, for better statistics (signal to noise ratio) in the images and to match the HESXRD repetition rate, the PLIF data were averaged with the result of an image every 0.5 s. Calibration measurements with known $\mathrm{CO}_{2}$ partial pressures were performed in order to correlate the detected PLIF signal to partial pressures. A schematic of the setup is shown in in Fig. 1.

\subsection{Reactor and sample preparation}

The reactor used for the experiment is based on the same design as the reactor described by Rijn, Ackermann et al., (2010). For the present experiment, a reactor dome, with a volume of $25 \mathrm{ml}$, made of sapphire for the transmission of both $\mathrm{X}$-ray and IR wavelengths, was used. Individual gas mass flow controllers (Bronkhorst EL-FLOW) were used for each gas 


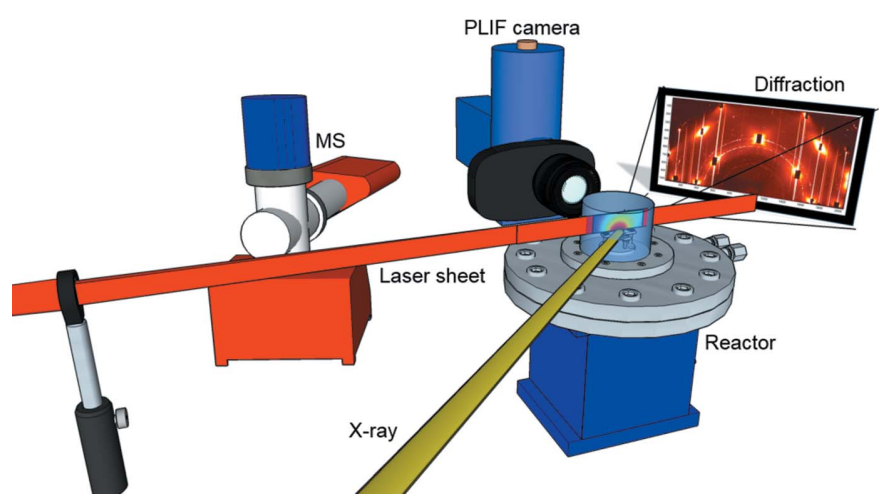

Figure 1

Schematic view of the experimental setup. The diffraction pattern from the (100) surface was detected in the forward direction with respect to the incoming X-rays. The laser sheet, with a wavelength of $2.7 \mu \mathrm{m}$, used to probe the $\mathrm{CO}_{2}$ gas phase, was sent through the reactor at an angle of approximately $45^{\circ}$ relative to the $\mathrm{X}$-rays. The camera for $\mathrm{CO}_{2}$ detection was positioned perpendicular to the laser sheet. In addition, an MS was connected to the outlet of the reactor to measure the global gas concentration.

with a capacity to individually flow up to $200 \mathrm{ml}_{\mathrm{n}} \min ^{-1}$. A pressure controller (Bronkhorst EL-PRESS) was attached to the outlet to keep the pressure constant throughout the experiments. The gas composition in the reactor was measured with a quadrupole MS (Pfeiffer PrismaPlus QMG220). The MS was connected to the outlet of the reactor via a 4 m-long tube (diameter $6 \mathrm{~mm}$ ), and a specially made automatic leak valve (LPM Leiden Probe Microscopy BV) was used to regulate the pressure in the MS. An implemented feedback system in the leak valve made it possible to control and keep the pressure constant in the MS, even though drastic changes in the gas flows were applied. The MS data were synchronized with the PLIF images using LabView while the HESXRD data and PLIF/MS were synchronized via timestamps in their individual data files.

The experimental setup is equipped with an ultra-high vacuum part where cleaning of the sample was carried out. The single crystal was cleaned by $\mathrm{Ar}^{+}$sputtering using an ion energy of $1.5 \mathrm{keV}$ in an Ar pressure of $1 \times 10^{-5}$ Torr. Oxygen treatments removed carbon contaminations by exposing the surface to $1 \times 10^{-6}$ Torr of $\mathrm{O}_{2}$ and heating the sample to $900 \mathrm{~K}$.

\section{Results}

To establish a better picture of the CO oxidation over $\mathrm{Pd}(100)$, we performed an operando surface-structure determination in combination with $\mathrm{CO}_{2}$ gas-phase visualization experiments. HESXRD data images acquired with a photon energy of $85 \mathrm{keV}$ cover a large part of the reciprocal space and reveal the $\mathrm{Pd}(100)$ surface structure. Simultaneously recorded PLIF images gained with a laser wavelength of $2.7 \mu \mathrm{m}$ show the $\mathrm{CO}_{2}$ gas-phase distribution above the $\operatorname{Pd}(100)$ surface. The surfacestructure and gas-phase images were matched, and the $\mathrm{CO}$ oxidation could be followed with an updating frequency of
$0.5 \mathrm{~s}$. In addition, an MS was positioned at the outlet of the reactor, sampling the global gas concentrations in the reactor.

To demonstrate the possibility of correlating the surface structure with a change in the gas-phase composition using PLIF and HESXRD, the catalytic activity was modulated by varying the gas ratios of $\mathrm{CO}$ and $\mathrm{O}_{2}$ at a constant temperature of $200{ }^{\circ} \mathrm{C}$ of the sample. The advantage of performing spatially resolved operando measurements with high temporal resolution is emphasized in Fig. 2, where the ignition of the catalyst can be followed in detail over time. At the start of the experiment, we exposed the $\mathrm{Pd}(100)$ to $6 \mathrm{mbar}$ of $\mathrm{CO}$ and 144 mbar of Ar but no $\mathrm{O}_{2}$, which generated an inactive and $\mathrm{CO}$ poisoned sample. The diffraction data recorded under this condition (Fig. 2a) shows Bragg reflections from the bulk and crystal truncation rods from the surface of the $\mathrm{Pd}(100)$, demonstrating that a well ordered metallic surface is present when no $\mathrm{CO}_{2}$ signal is detected in the gas phase over the surface (Fig. 2b). To activate the $\operatorname{Pd}(100)$ catalyst, the gas composition was changed to reaction conditions by adding $24 \mathrm{mbar}$ of $\mathrm{O}_{2}$ simultaneously as the $\mathrm{Ar}$ pressure was decreased to 120 mbar in order to maintain a total pressure of 150 mbar constant. Using these reaction conditions, we found that a sample temperature of $200{ }^{\circ} \mathrm{C}$ was sufficient to bring the reaction into the mass-transfer limited (MTL) regime where a characteristic boundary layer of $\mathrm{CO}_{2}$ was observed (Figs. $2 d$ and $2 f$ ), consistent with previous PLIF studies of the gas phase in the MTL regime (Zetterberg et al., 2015). The boundary layer of the $\mathrm{CO}_{2}$ product species accumulated around the surface inhibits the $\mathrm{CO}$ molecules reaching the surface and the reaction becomes CO-diffusion limited (Matera \& Reuter, 2012). As a consequence of the boundary layer formation, the gas composition within the boundary layer is significantly different compared with the rest of the reactor, resulting in a more oxidizing environment above the surface in this regime (Blomberg et al., 2016). At this early stage in the MTL regime, a well ordered oxidized surface is not yet formed and the metallic phase of the surface is still detected in the HESXRD image (Fig. 2c). We conclude that, approximately $2 \mathrm{~s}$ after ignition of the catalyst (Fig. 2e), a diffraction pattern with surface truncation rods corresponding to the $(\sqrt{5} \times \sqrt{5})$-R2 $27^{\circ}$ surface oxide (Todorova et al., 2003; Gustafson et al., 2014; Shipilin et al., 2014) could be observed. After that, the surface oxide rods become more intense and the $\mathrm{CO}_{2}$ boundary layer is continuously observed for the remaining time of the experiment.

For the purpose of deactivating the catalyst, the flow of oxygen was turned off, and the same initial conditions of the experiment were present in the reactor. As the $\mathrm{CO}_{2}$ PLIF signal dropped, the surface oxide was reduced and could not be observed in the diffraction data when the $\mathrm{CO}_{2}$ signal close to the sample was approximately zero.

To correlate the presence of a surface oxide with the $\mathrm{CO}_{2}$ concentration above the catalyst surface, the intensity variation of the truncation rods originating from the $(\sqrt{5} \times \sqrt{5})$ $\mathrm{R} 27^{\circ}$ surface oxide during the $\mathrm{CO}$ oxidation experiment, is studied more in detail and compared with the $\mathrm{CO}_{2}$ PLIF signal extracted from an area of $1.65 \mathrm{~mm}^{2}, 0.3 \mathrm{~mm}$ above the surface 


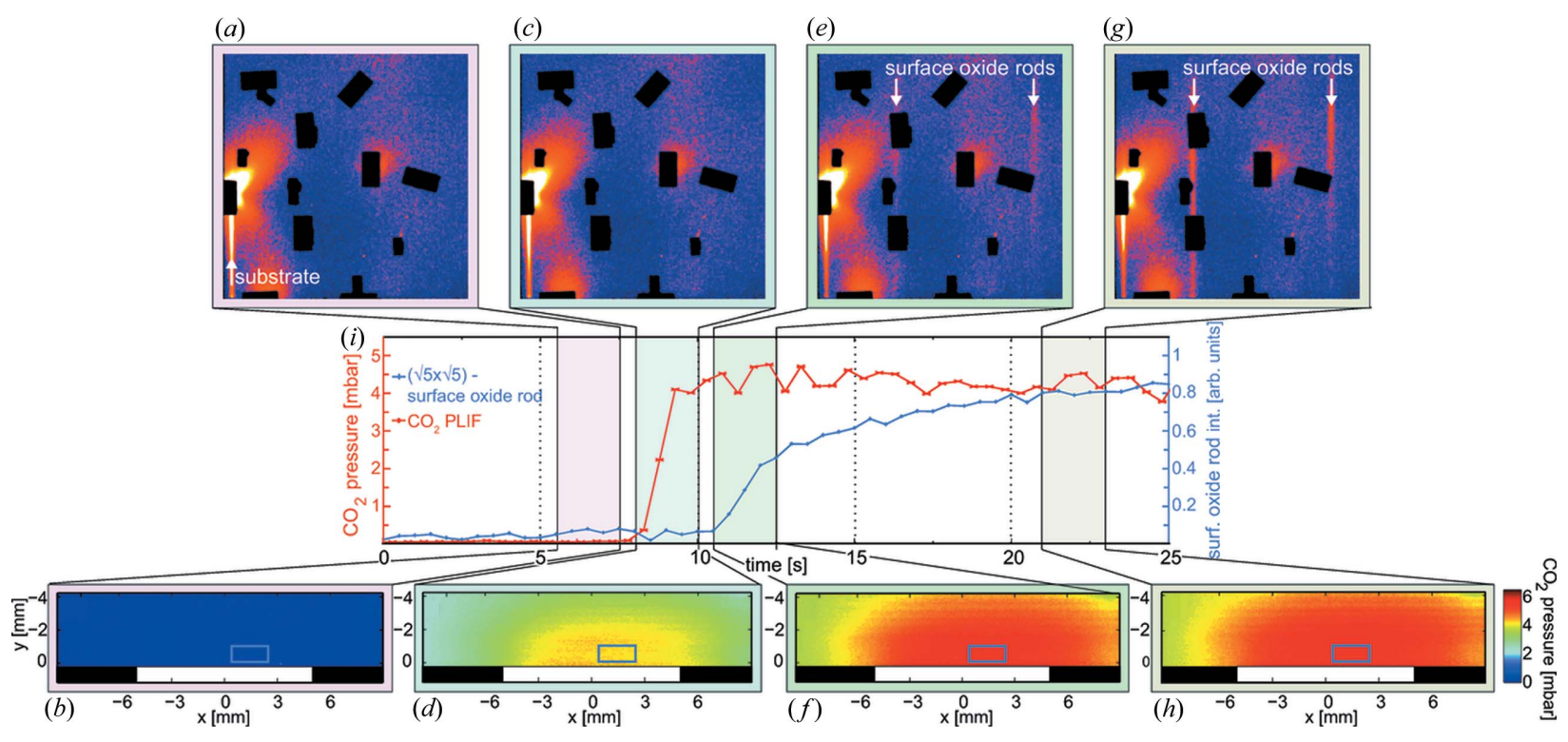

Figure 2

CO oxidation over Pd(100). Each image (HESXRD and PLIF) is averaged over $2.5 \mathrm{~s}$ (shown as a colored area in the graph) for better statistics. The graph in $(i)$ shows how the $\mathrm{CO}_{2}$ PLIF signal, extracted from the area marked with a blue rectangle in $(b),(d),(f)$ and $(h)$, together with the intensity of the surface-oxide rod, changes over time. A linear background is subtracted from the plotted HESXRD surface-oxide rod intensity. The data are plotted with an updating frequency of $0.5 \mathrm{~s}$. (a) HESXRD image of an inactive sample. A surface truncation rod from the (100) substrate is detected (lower left corner), indicating that a metallic surface is present. $(b) \mathrm{No} \mathrm{CO}_{2}$ is detected in the gas phase above the sample surface. (c) A metallic surface is detected in the HESXRD data. (d) PLIF image showing the ignition of the reaction as the sample becomes active. (e) After the sample has been active for about $2.5 \mathrm{~s}$, superstructure rods from the diffraction of the $(\sqrt{5} \times \sqrt{5})$-R $27^{\circ}$ surface oxide (white arrows) appear in the diffraction pattern. $(f)$ As the sample becomes active, a prominent boundary layer of $\mathrm{CO}_{2}$ is detected using PLIF. $(g)$ After additional reaction time, the $(\sqrt{5} \times \sqrt{5})$ - $\mathrm{R} 27^{\circ}$ surface-oxide diffraction pattern intensifies. (h) PLIF images showing that the $\mathrm{CO}_{2}$ boundary layer is still present over the surface.

(Fig. 3a). The graph illustrates that the temporal resolution with which we can follow the reaction (HESXRD $2 \mathrm{~Hz}$, PLIF $10 \mathrm{~Hz}$ ) is sufficient to monitor the ignition of the reaction both in the gas phase and through the surface reconstruction. The sudden increase in $\mathrm{CO}_{2}$ production upon sample ignition is easily captured by PLIF though it is clear that the dynamics on the surface forming a well ordered surface oxide is slower. The growth can be followed by studying the intensity of the diffraction rod from the surface oxide, which shows that it takes $2.5 \mathrm{~s}$ before any sign of the oxide can be detected. Our results indicate that a metallic surface is present at the ignition of the reaction, but we cannot exclude small domains that are not visible in the diffraction pattern of the $(\sqrt{5} \times \sqrt{5})$-R2 $27^{\circ}$ structure present at the surface. The intensity of the surface-oxide rods increases slowly for around $10 \mathrm{~s}$ during the experiment, consistent with a growth of the $(\sqrt{5} \times \sqrt{5})-\mathrm{R} 27^{\circ}$ islands and is interpreted as the surface being, to a large extent, covered with the $(\sqrt{5} \times \sqrt{5})-\mathrm{R} 27^{\circ}$ surface oxide $10 \mathrm{~s}$ after ignition.

An MS attached via a 4 m-long (diameter $6 \mathrm{~mm}$ ) gas line at the outlet of

Figure 3 the reactor enabled us to achieve information on the global gas concentrations in the reactor. For the purpose of evaluating the gas detecting techniques used in our setup, we compared the MS signal with the PLIF signal (Fig. 3b). The PLIF signal originates from the $\mathrm{CO}_{2}$ gas molecules that are

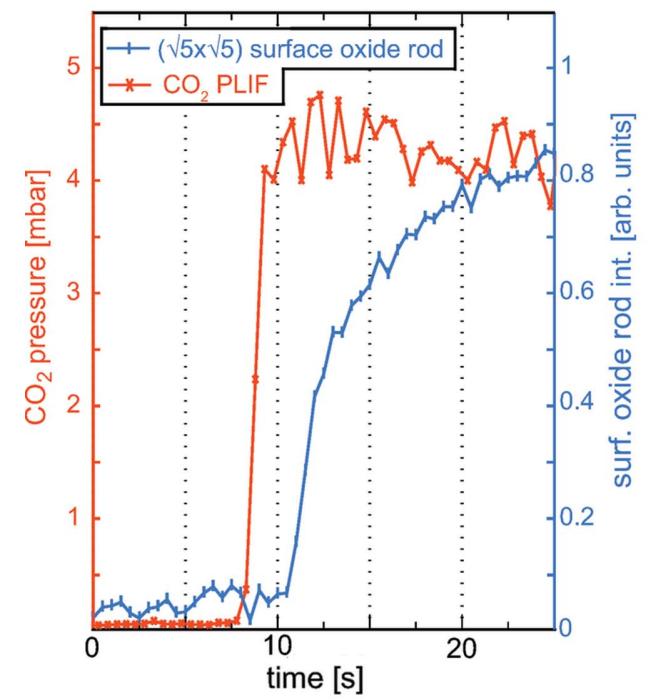

(a)

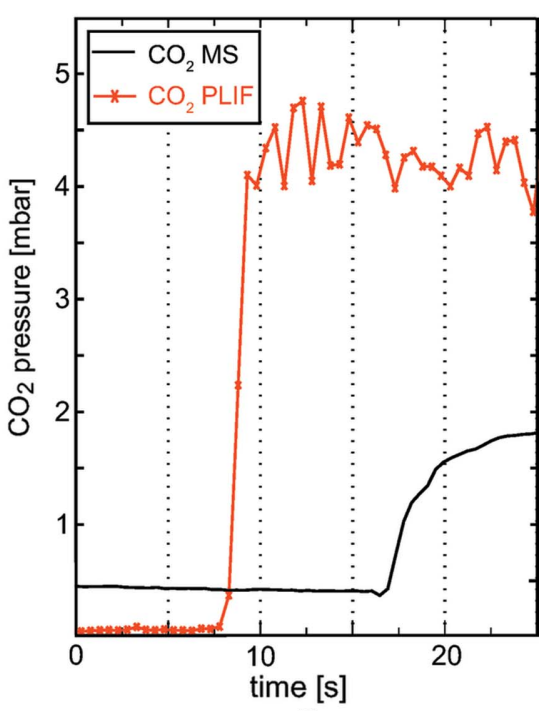

(b)

Data extracted from a series of images recorded during the experiment shown in Fig. 2. (a) The total intensity of the superstructure rod from the $(\sqrt{5} \times \sqrt{5})-\mathrm{R} 27^{\circ}$ surface oxide plotted together with the $\mathrm{CO}_{2}$ PLIF signal extracted from the blue rectangle indicated in the PLIF images in Fig. 2, allowing for a better correlation between the surface structure and $\mathrm{CO}_{2}$ production. $(b)$ The same PLIF $\mathrm{CO}_{2}$ signal as in $(a)$ plotted together with the $\mathrm{CO}_{2}$ MS signal. 
detected almost instantaneously after they desorb from the active surface. Using the PLIF signal as a reference, we determined the response time of the MS to be $9 \mathrm{~s}$. By comparing the PLIF and MS signals, we could also conclude that a $\mathrm{CO}_{2}$ partial pressure of around $4-4.5 \mathrm{mbar}$ was measured just above the surface in the MTL regime, while surprisingly, only 2 mbar was detected by the MS during the same period. Neither of the detected $\mathrm{CO}_{2}$ concentrations indicate a $100 \%$ conversion of $\mathrm{CO}$, but the MS shows a significantly lower concentration than that detected close to the surface using PLIF. The large discrepancy between the measured $\mathrm{CO}_{2}$ partial pressures can be explained by the global detection of the gas by MS that suffers from the complex gasflow configuration in the reactor (Matera et al., 2014), which smears out the signal as compared with the $\mathrm{CO}_{2}$ signal detected just above the surface by PLIF.

\section{Conclusions and discussion}

The CO oxidation over $\mathrm{Pd}(100)$ has previously been studied in great detail, but the question remains whether it is the metallic or oxidized surface that is the most active phase. Herein, we report on the first operando measurement where HESXRD, used for surface-structure determination, is combined with PLIF to detect the $\mathrm{CO}_{2}$ gas in the vicinity of a catalyst surface. PLIF provides an image of the $\mathrm{CO}_{2}$ almost instantaneously as the $\mathrm{CO}_{2}$ desorbs from the surface, which enables a correlation to be made between the active surface structure and the gasphase environment.

PLIF images reveal a boundary layer of $\mathrm{CO}_{2}$ close to the surface when the $\mathrm{Pd}(100)$ model catalyst is in the MTL regime, resulting in a gas composition in the vicinity of the surface that is significantly different to the concentration detected by the MS at the outlet of the reactor. We conclude that the oxidizing environment, caused by the boundary layer, oxidizes the surface to a large extent, which implies that the catalytic activity of the sample itself affects the surface structure. Our findings show that the surface is not fully oxidized when the ignition of the catalyst occurs, but we cannot exclude that oxidized islands, not detectable with the diffraction, are present on the surface. After several seconds in the highly active regime, however, a strong signal from the surface oxide is detected in the diffraction pattern. Moreover, our data confirm that the response time of the MS is critical when establishing a correlation between surface structure and activity. The delay time of the MS, which is dependent on both the experimental setup and conditions applied, may introduce an uncertainty when measuring and comparing the catalytic activity of a catalyst at different setups, even though identical conditions have been applied. Fortunately, in the present setup, it is possible to measure the response time for the MS by comparing the signals from the MS and PLIF, but to estimate the time delay for the gas detection by MS is generally not trivial. The advantage of using PLIF to complement MS is also demonstrated by extracting the partial pressure of $\mathrm{CO}_{2}$, located above the sample surface, which differs significantly from the pressure detected by the MS at the reactor outlet.
In the present study, our measurements were limited by a constant temperature as a result of the critical alignment of the X-rays that should have an incident angle of $0.04^{\circ}$ on the sample surface and temperature variation may misalign the sample. It would therefore be interesting to combine PLIF with transmission X-ray diffraction (Reikowski et al., 2017; Acciarri et al., 1996), where X-rays penetrate the sample and as a result are less sensitive to temperature variations. The transmission mode of the $\mathrm{X}$-rays would also generate a smaller footprint of the X-ray on the sample surface, which opens up for better spatially resolved surface structure measurements. In the meantime, our results show a unique possibility to relate the activity of the catalyst to the surface structure by combining PLIF and HESXRD. These observations were possible because of the high temporal and spatial resolution in conjunction with immediate detection of desorbed $\mathrm{CO}_{2}$ molecules from the active surface.

\section{References}

Acciarri, M. et al. (1996). Phys. Lett. B, 371, 137-148.

Ackermann, M. D., Pedersen, T. M., Hendriksen, B. L. M., Robach, O., Bobaru, S. C., Popa, I., Quiros, C., Kim, H., Hammer, B., Ferrer, S. \& Frenken, J. W. M. (2005). Phys. Rev. Lett. 95, 255505.

Alwahabi, Z. T., Zetterberg, J., Li, Z. S. \& Aldén, M. (2007). Eur. Phys. J. D, 42, 41-47.

Blomberg, S., Hoffmann, M. J., Gustafson, J., Martin, N. M., Fernandes, V. R., Borg, A., Liu, Z., Chang, R., Matera, S., Reuter, K. \& Lundgren, E. (2013). Phys. Rev. Lett. 110, 117601.

Blomberg, S., Zhou, J. F., Gustafson, J., Zetterberg, J. \& Lundgren, E. (2016). J. Phys. Condens. Matter, 28, 453002.

Chen, M. S., Cai, Y., Yan, Z., Gath, K. K., Axnanda, S. \& Goodman, D. W. (2007). Surf. Sci. 601, 5326-5331.

Ertl, G., Knozinger, H. \& Weitkamp, J. (1997). Handbook of Heterogeneous Catalysis. Weinheim: Wiley-VCH.

Freund, H. J., Meijer, G., Scheffler, M., Schlögl, R. \& Wolf, M. (2011). Angew. Chem. Int. Ed. 50, 10064-10094.

Gao, F., Wang, Y., Cai, Y. \& Goodman, D. W. (2009). J. Phys. Chem. $C, \mathbf{1 1 3}, 174-181$.

Gao, F., Wang, Y. L. \& Goodman, D. W. (2010). J. Phys. Chem. C, 114, 6874.

Goldenstein, C. S., Miller, V. A. \& Hanson, R. K. (2015). Appl. Phys. $B$, 120, 185-199.

Gustafson, J., Mikkelsen, A., Borg, M., Andersen, J. N., Lundgren, E., Klein, C., Hofer, W., Schmid, M., Varga, P., Köhler, L., Kresse, G., Kasper, N., Stierle, A. \& Dosch, H. (2005). Phys. Rev. B, 71, 115442.

Gustafson, J., Shipilin, M., Zhang, C., Stierle, A., Hejral, U., Ruett, U., Gutowski, O., Carlsson, P. A., Skoglundh, M. \& Lundgren, E. (2014). Science, 343, 758-761.

Gustafson, J., Westerström, R., Mikkelsen, A., Torrelles, X., Balmes, O., Bovet, N., Andersen, J. N., Baddeley, C. J. \& Lundgren, E. (2008). Phys. Rev. B, 78, 045423.

Hammer, B. (2006). Top. Catal. 37, 3-16.

Head, A. R., Karslıoğlu, O., Gerber, T., Yu, Y., Trotochaud, L., Raso, J., Kerger, P. \& Bluhm, H. (2017). Surf. Sci. 665, 51-55.

Hejral, U., Muller, P., Balmes, O., Pontoni, D. \& Stierle, A. (2016). Nat. Commun. 7, 10964.

Hejral, U., Muller, P., Shipilin, M., Gustafson, J., Franz, D., Shayduk, R., Rutt, U., Zhang, C., Merte, L. R., Lundgren, E., Vonk, V. \& Stierle, A. (2017). Phys. Rev. B, 96, 195433. 
Hendriksen, B. L. M., Ackermann, M. D., van Rijn, R., Stoltz, D., Popa, I., Balmes, O., Resta, A., Wermeille, D., Felici, R., Ferrer, S. \& Frenken, J. W. M. (2010). Nat. Chem. 2, 730-734.

King, A., Beckmann, F., Muller, M., Schreyer, A., Schell, N. \& Fischer, T. (2014). Mechanical Stress Evaluation by Neutrons and Synchrotron Radiation VI, Vol. 772, pp. 57-61. Trans Tech Publications.

Kirby, B. J. \& Hanson, R. K. (2002). Appl. Opt. 41, 1190-1201.

Lundgren, E., Zhang, C., Merte, L. R., Shipilin, M., Blomberg, S., Hejral, U., Zhou, J. F., Zetterberg, J. \& Gustafson, J. (2017). Acc. Chem. Res. 50, 2326-2333.

Matera, S., Maestri, M., Cuoci, A. \& Reuter, K. (2014). ACS Catal. 4, 4081-4092.

Matera, S. \& Reuter, K. (2012). J. Catal. 295, 261-268.

Mavrikakis, M., Hammer, B. \& Nørskov, J. K. (1998). Phys. Rev. Lett. 81, 2819-2822.

Reikowski, F., Wiegmann, T., Stettner, J., Drnec, J., Honkimäki, V., Maroun, F., Allongue, P. \& Magnussen, O. M. (2017). J. Phys. Chem. Lett. 8, 1067-1071.

Rijn, R. van, Ackermann, M. D., Balmes, O., Dufrane, T., Geluk, A., Gonzalez, H., Isern, H., de Kuyper, E., Petit, L., Sole, V. A., Wermeille, D., Felici, R. \& Frenken, J. W. M. (2010). Rev. Sci. Instrum. 81, 014101.

Rijn, R. van, Balmes, O., Felici, R., Gustafson, J., Wermeille, D., Westerström, R., Lundgren, E. \& Frenken, J. W. M. (2010). J. Phys. Chem. C, 114, 6875-6876.

Rupprechter, G. \& Weilach, C. (2007). Nano Today 2, 20-29.
Shipilin, M., Gustafson, J., Zhang, C., Merte, L. R. \& Lundgren, E. (2016). Phys. Chem. Chem. Phys. 18, 20312-20320.

Shipilin, M., Hejral, U., Lundgren, E., Merte, L. R., Zhang, C., Stierle, A., Ruett, U., Gutowski, O., Skoglundh, M., Carlsson, P. A. \& Gustafson, J. (2014). Surf. Sci. 630, 229-235.

Somorjai, G. A. \& Li, Y. (2010). Introduction to Surface Chemistry and Catalysis, 2nd ed. Hoboken: Wiley-Blackwell.

Tinnemans, S. J., Mesu, J. G., Kervinen, K., Visser, T., Nijhuis, T. A., Beale, A. M., Keller, D. E., van der Eerden, A. M. J. \& Weckhuysen, B. M. (2006). Catal. Today, 113, 3-15.

Todorova, M., Lundgren, E., Blum, V., Mikkelsen, A., Gray, S., Gustafson, J., Borg, M., Rogal, J., Reuter, K., Andersen, J. N. \& Scheffler, M. (2003). Surf. Sci. 541, 101-112.

Toyoshima, R. \& Kondoh, H. (2015). J. Phys. Condens. Matter, 27, 083003.

Westerström, R., Gustafson, J., Resta, A., Mikkelsen, A., Andersen, J. N., Lundgren, E., Seriani, N., Mittendorfer, F., Schmid, M., Klikovits, J., Varga, P., Ackermann, M. D., Frenken, J. W. M., Kasper, N. \& Stierle, A. (2007). Phys. Rev. B, 76, 155410.

Zetterberg, J., Blomberg, S., Gustafson, J., Evertsson, J., Zhou, J., Adams, C. E., Carlsson, P. A., Aldén, M. \& Lundgren, E. (2015). Nat. Commun. 6, 7076.

Zetterberg, J., Blomberg, S., Gustafson, J., Sun, Z. W., Li, Z. S., Lundgren, E. \& Alden, M. (2012). Rev. Sci. Instrum. 83, 053104.

Zhou, J. F., Pfaff, S., Lundgren, E. \& Zetterberg, J. (2017). Appl. Phys. $B, \mathbf{1 2 3}, 3$. 